\title{
Neutrophil extracellular trap production contributes to pathogenesis in SIV-infected nonhuman primates
}

\author{
Ranjit Sivanandham, ${ }^{1,2}$ Egidio Brocca-Cofano, ${ }^{1,2}$ Noah Krampe, ${ }^{1}$ Elizabeth Falwell, ${ }^{1,3}$ Sindhuja Murali Kilapandal Venkatraman,, \\ Ruy M. Ribeiro, ${ }^{4}$ Cristian Apetrei, ${ }^{1,3,5}$ and Ivona Pandrea ${ }^{1,2,5}$ \\ 'Center for Vaccine Research, ${ }^{2}$ Department of Pathology, and ${ }^{3}$ Department of Microbiology and Molecular Cenetics, School of Medicine, University of Pittsburgh, Pittsburgh, Pennsylvania, USA. \\ ${ }^{4}$ Laboratorio de Biomatematica, Faculdade de Medicina da Universidade de Lisboa, Lisbon, Portugal. ${ }^{5}$ Department of Infectious Diseases and Microbiology, Graduate School of Public Health, \\ University of Pittsburgh, Pittsburgh, Pennsylvania, USA.
}

\begin{abstract}
Neutrophil extracellular traps (NETs) are involved in the pathogenesis of many infectious diseases, yet their dynamics and impact on HIV/SIV infection have not yet been assessed. We hypothesized that SIV infection and the related microbial translocation trigger NET activation and release (NETosis), and we investigated the interactions between NETs and immune cell populations and platelets. We compared and contrasted the levels of NETs between SIV-uninfected, SIV-infected, and SIVinfected antiretroviral-treated nonhuman primates. We also cocultured neutrophils from these animals with either peripheral blood mononuclear cells or platelets. Increased NET production was observed throughout SIV infection. In chronically infected animals, NETs were found in the gut, lung, and liver, and in the blood vessels of kidney and heart. Antiretroviral therapy (ART) decreased NETosis, albeit above preinfection levels. NETs captured CD4+ and CD8+ $T$ cells, B cells, and monocytes, irrespective of their infection status, potentially contributing to the indiscriminate generalized immune cell loss characteristic to HIV/SIV infection, and limiting the CD4 ${ }^{+} \mathrm{T}$ cell recovery under ART. By capturing and facilitating aggregation of platelets, and through expression of increased tissue factor levels, NETs may also enhance HIV/SIV-related coagulopathy and promote cardiovascular comorbidities.
\end{abstract}

\section{Introduction}

Neutrophils are central to the innate immune system, being involved in the defense against bacteria and fungi (1), and even against viruses, as recently reported (2). In addition to phagocytizing and killing microorganisms, neutrophils can control infections through generation of extracellular chromatin fibers called neutrophil extracellular traps (NETs) (3). Neutrophils that release NETs develop a unique cellular morphology with decondensed nuclei that ultimately lose their DNA (4). NETs are complex structures consisting of chromatin and proteins, such as lactoferrin, myeloperoxidase (MPO), histones, and neutrophil elastase (NE) (5). In vitro-generated NETs are long, thin-stranded, web-like extracellular fibers (1). NETs with a thicker morphology were identified in vivo in the gut, liver, skin, and lung in numerous diseases $(4,6,7)$.

NETs can capture bacteria (1), fungi (5), and viruses, promoting their elimination (8). For example, HIV-1 stimulates neutrophils to produce NETs, through TLR7/TLR8. NETs can then capture HIV- 1 virions and inactivate them via MPO and $\alpha$-defensins (8). NETs are not always beneficial: they promote thrombosis (9), being involved in the pathogenesis of cardiovascular and autoimmune diseases. In cancers, NETs facilitate metastasis by sequestrating circulating tumor cells (10).

Authorship note: RS and EBC contributed equally to this work. Conflict of interest: The authors have declared that no conflict of interest exists. License: Copyright 2018, American Society for Clinical Investigation. Submitted: December 20, 2017; Accepted: August 30, 2018. Reference information: J Clin Invest. 2018;128(11):5178-5183. https://doi.org/10.1172/JCI99420.
We thus studied the dynamics and functions of NETs during SIV infection, to assess their contribution to disease progression and comorbidities. We report that (a) NET production increases throughout untreated SIV infection, being only partially reduced by ART, (b) NETs may contribute to the indiscriminate depletion of immune cells that are not direct virus targets, and to the incomplete $\mathrm{CD}^{+}{ }^{+} \mathrm{T}$ cell restoration observed in HIV-infected subjects on ART, and (c) NETosis may promote thrombosis in the thrombocytopenic environment of HIV/SIV infections by capturing platelets and expressing tissue factor (TF).

\section{Results and Discussion}

We assessed the role of NETs in the pathogenesis of HIV/SIV infection in 37 pigtailed macaques (Macaca nemestrina; PTMs). Ten PTMs were inoculated with SIVsab92018 and used to assess NET dynamics during SIV infection. The impact of ART on NET formation was evaluated in 12 additional SIVsab-infected PTMs receiving coformulated ART for 10 months, initiated at 50 days postinfection (dpi), and virologically suppressed below the detection limit (30 vRNA copies/ $\mathrm{ml}$ ). Ten uninfected PTMs housed and followed in the same conditions as the SIV-infected ones were used as controls. Five additional uninfected PTMs were used for apoptosis studies. Peripheral blood mononuclear cells (PBMCs), neutrophils, and platelets were isolated from blood collected either prior to infection or at critical time points after infection and treatment. Tissues from 25 chronically SIV-infected PTMs from other studies were used for histology.

Previous reports showed that neutrophils isolated from uninfected subjects can release NETs that capture HIV (8), yet NET production in HIV-infected subjects has never been demonstrated. Fur- 

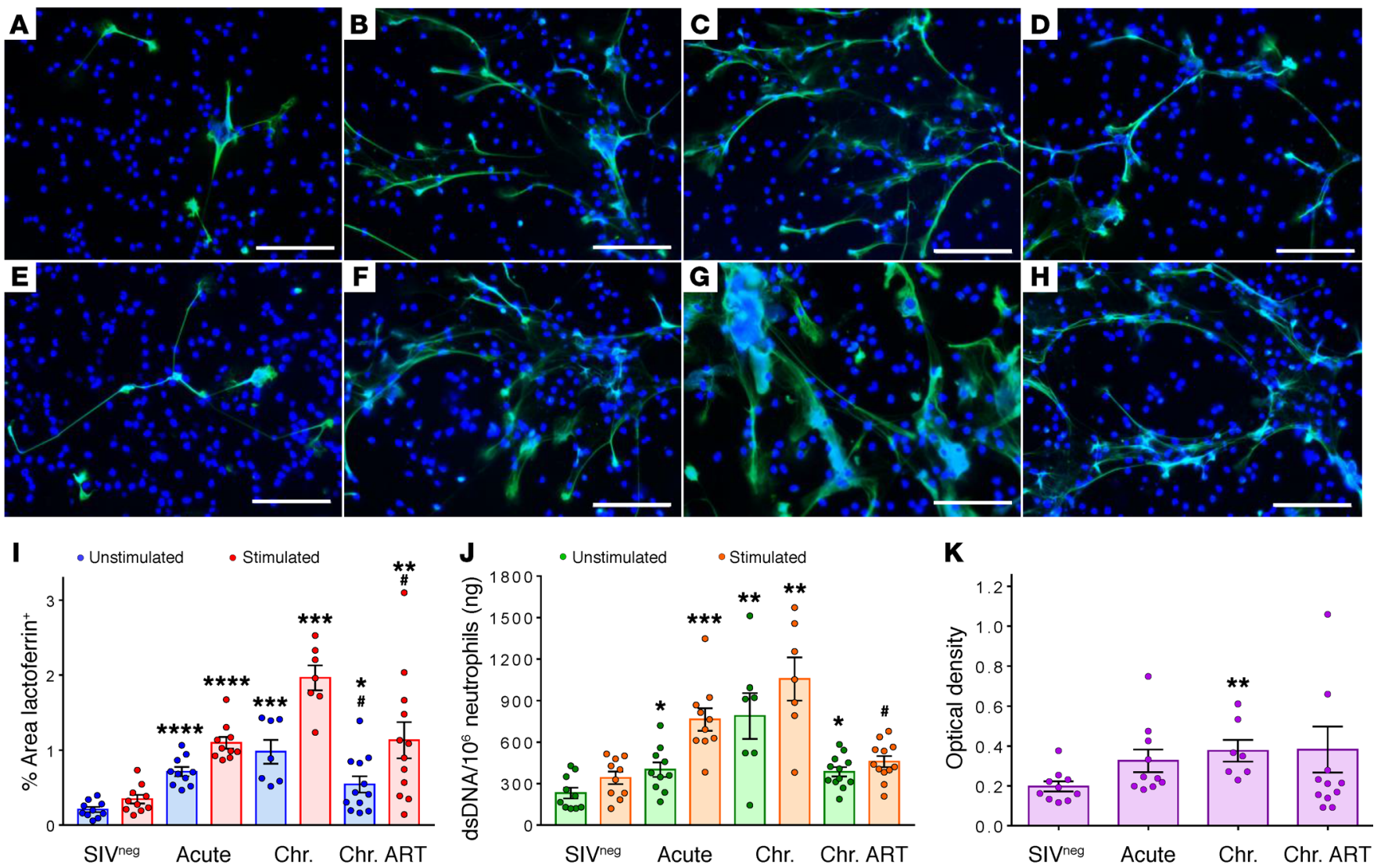

Figure 1. NET dynamics in SIV infection. NET production in unstimulated (A-D) and stimulated PMNs (E-H): prior to infection (SIVneg; $n=10 ; \mathbf{A}$ and E), during acute SIV infection (Acute; $n=10 ; \mathbf{B}$ and $\mathbf{F}$ ), during chronic infection (Chr.; $n=7 ; \mathbf{C}$ and $\mathbf{G}$ ), and in SIV-infected PTMs receiving ART (Chr. ART; $n=12 ; \mathbf{D}$ and $\mathbf{H}$ ). NETs were identified by immunohistochemical staining for lactoferrin (green); neutrophils were stained with DAPI (blue). Quantitative image analyses showing the percentage of the area positive for lactoferrin as a NET marker in unstimulated (blue) and stimulated (red) samples (I). Picogreen dsDNA quantification in unstimulated (green) and stimulated (orange) samples (J). Dynamics of NETs, in plasma, assessed by ELISA (K). Scale bars: $100 \mu \mathrm{m}$. A 2-tailed Mann-Whitney $U$ test was used for statistical analyses, significance being defined as compared with baseline preinfection values after Bonferroni's correction for multiple comparisons: ${ }^{*} P<0.05,{ }^{* *} P<0.01,{ }^{* *} P<0.001$, ${ }^{* * *} P<0.0001$, or to chronic infection ${ }^{\#} P<0.05,{ }^{\# \#} P<0.01$. Actual $P$ values are shown in Supplemental Table 1 .

thermore, the dynamics of NET production during HIV infection, and the impact of ART on NETosis, are so far unknown. To address these questions, neutrophils isolated from untreated and treated SIV-infected PTMs were incubated in the presence or absence of PMA, and stained for essential NET markers (Supplemental Figure 1; supplemental material available online with this article; https://doi. org/10.1172/JCI99420DS1). This strategy allowed identification of the characteristic NET filaments, in which neutrophil-derived proteins such as histone H3 (Supplemental Figure 1A), MPO (Supplemental Figure 1B), lactoferrin (Supplemental Figure 1C), or NE (Supplemental Figure 1D) colocalized with extracellular DNA (DAPI).

To determine the effect of gram-positive and gram-negative bacteria on NETosis, we incubated neutrophils with Staphylococcus aureus (ref. 1 and Supplemental Figure 2A), or Escherichia coli (Supplemental Figure 2B). Both conditions elicited NET formation, with bacteria being trapped in the NETs. We also observed SIV virion capture in the NETs (Supplemental Figure 2C), in agreement with studies showing similar HIV trapping (8). This suggests that indeed, neutrophils from HIV-infected subjects and SIV-infected nonhuman primates (NHPs) are particularly prone to NET overproduction through excessive stimulation by the virus, and by bacterial products translocated from the gut (11).
We monitored NET dynamics by comparing and quantifying NETs at critical time points before and after SIV infection, using immunofluorescence staining and picogreen dsDNA quantification. In SIV-uninfected NHPs, both unstimulated and stimulated neutrophils produced minimal levels of thin NETs (Figure 1, A and E, and Supplemental Figure 3). Neutrophils isolated during acute SIV infection (14 dpi) showed a dramatic increase of NET production (Figure 1, B and F). Because this early NET increase occurred prior to the major alterations in gut integrity, we concluded that SIV itself contributes to NET formation (Supplemental Figure 2C). A progressive and significant increase of NET production by neutrophils isolated throughout the follow-up (90 dpi) (Figure 1, C and G) was documented by immunofluorescence staining (Figure 1I) and picogreen dsDNA quantification (Figure 1J) in both unstimulated and stimulated neutrophils. The increased NETosis in unstimulated neutrophils isolated during chronic infection likely occurs as a consequence of SIV-induced severe gut damage and microbial translocation, which release potent NET triggers (11). ART suppressed the virus and reduced NET production by isolated neutrophils, but did not normalize it to preinfection levels in all the SIV-infected PTMs (Figure 1, D and $\mathrm{H}-\mathrm{J}$ ). This is likely due to incomplete healing of the intestine in virus-suppressed macaques, leading to incomplete res- 

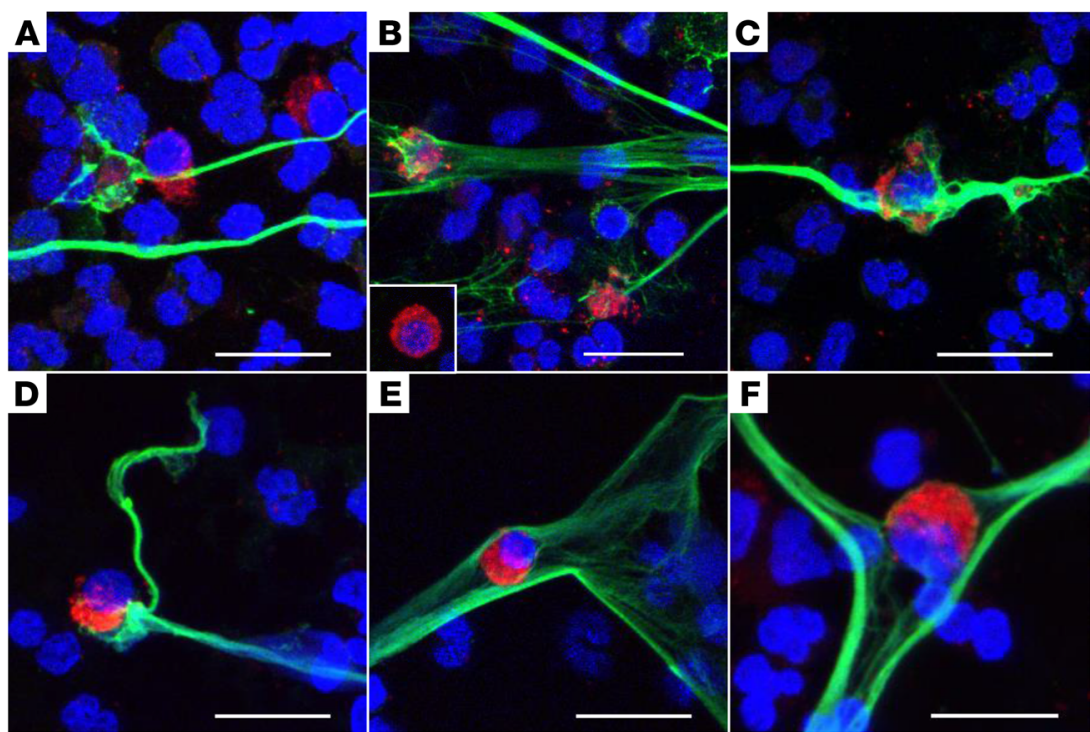

$\mathbf{G}$
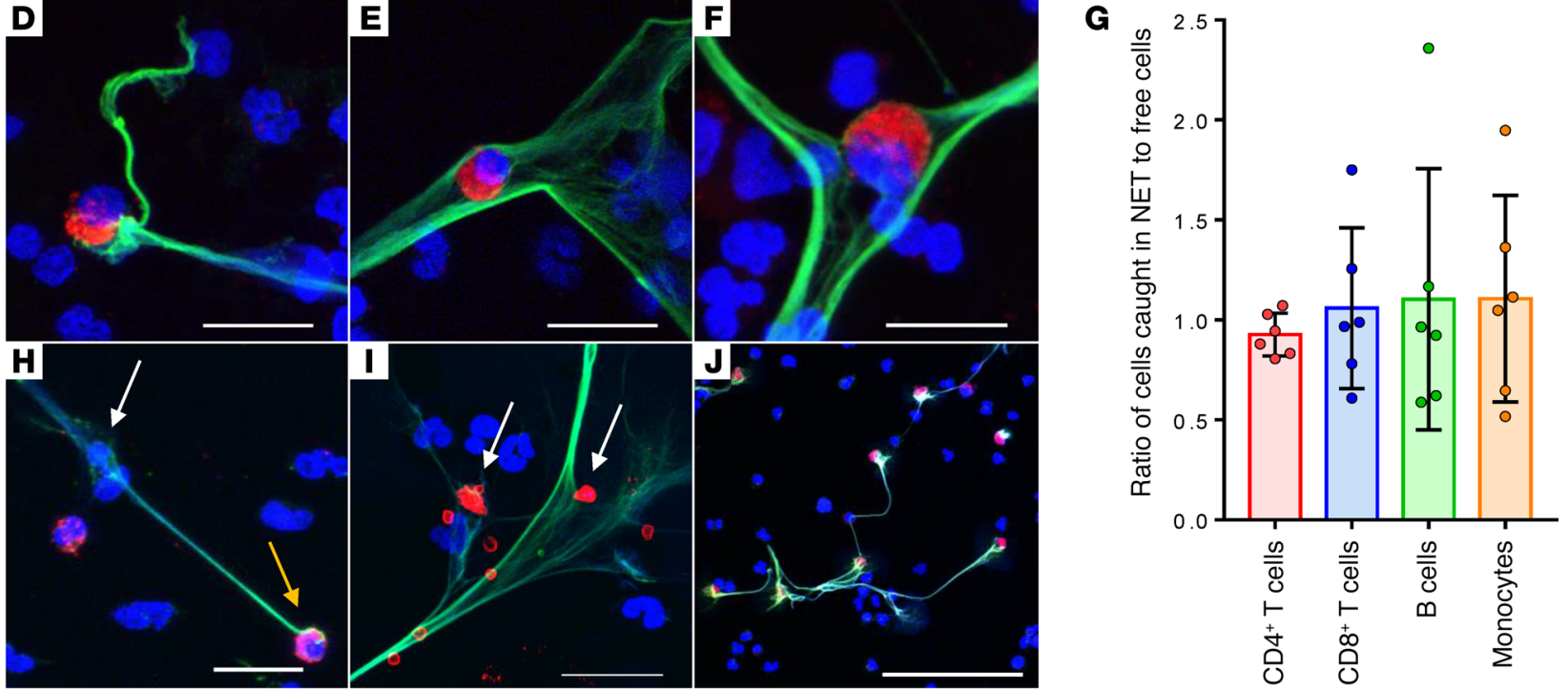

Figure 2. Key collateral damage associated with excessive NET formation during SIV infection. CD4+ $T$ cell (red) capture (A) and destruction (B) in the NETs; the inset in B shows a free $\mathrm{CD} 4^{+} \mathrm{T}$ cell for comparison. CD4 ${ }^{+} \mathrm{T}$ cell capture and destruction in chronically infected PTMs on ART (C). Other immune cells (red) caught in NETs include CD8 ${ }^{+}$T cells (D), B cells (E), and monocytes (F). The ratios of cells caught in NETs versus those outside of NETs were similar for the various cell types, indicating no preferential capture $(n=6)(\mathbf{C})$. Combined SIV RNAScope (red), lactoferrin immunofluorescence (green), and nuclear staining with DAPI (blue) showed both infected (orange arrow) and uninfected (white arrow) cells caught in NETs (H). Individual platelets (red circles) caught in NETs (green), and aggregated platelets (red) in the NETs (arrow) (I). Expression of TF (red) by neutrophils undergoing NETosis and their NETs (green), and lack of TF expression by normal neutrophils (blue) (J). Stimulated neutrophils shown in A-F, $\mathbf{H}$, and I; unstimulated neutrophils shown in J. Scale bars: $20 \mu \mathrm{m}$ (A-I), $100 \mu \mathrm{m}(\mathrm{J})$.

olution of microbial translocation (12). The dynamics of NETosis were confirmed in vivo with a NET ELISA on plasma samples from the same SIV-infected PTMs (ref. 13 and Figure 1K).

$\mathrm{CD}^{+}{ }^{+} \mathrm{T}$ cell depletion, the hallmark of HIV/SIV infection, occurs mainly by direct virus cytopathic effect and bystander effects of excessive immune activation (14). However, these mechanisms do not fully explain the magnitude of $\mathrm{CD} 4^{+} \mathrm{T}$ cell loss observed during SIV/HIV infection. We hypothesized that immune cell trapping in the NETs may also account for $\mathrm{CD}^{+}{ }^{+} \mathrm{T}$ cell loss during HIV/SIV infection. We therefore incubated PBMCs with neutrophils from SIV-infected PTMs in the presence or absence of a NET stimulus and observed $\mathrm{CD}^{+} \mathrm{T}$ cell capture (Figure $2 \mathrm{~A}$ and Supplemental Figure 4) and destruction (Figure 2, B and C) in the NETs, as demonstrated by the $\mathrm{T}$ cell morphological changes (membrane bleb formation, cell membrane disintegration, nuclei irregularities, and fragmentation) (Figure 2, B and C). These changes involved only the $\mathrm{CD}^{+} \mathrm{T}$ cells caught in the NETs, and not those free in media (Figure 2B, inset).

To confirm that cell capture by NETs is indeed deadly, we performed a series of experiments in which we first incubated PBMCs and neutrophils isolated from uninfected PTMs in the presence or absence of NET stimuli and then treated cultures with nucleases, to break the NETs (Figure 3, A-D). Only the cells recovered from the stimulated cell cultures showed increases in annexin $\mathrm{V}$ apoptosis marker (Figure 3, C and D), in accordance with the NET presence in cultures (Figure 1E), as opposed to minimal NET production in unstimulated samples (Figure 1A). Cell death in the NETs was also directly confirmed by in situ quantification of active caspase-3 IHC staining in these cell cultures from uninfected animals (Figure 3, E and F, and Supplemental Figure 5), as well as in cocultures of nonstimulated PBMCs and neutrophils from SIV-infected PTMs (Figure 3, G and H).

We thus directly proved that capture by the NETs may represent a previously unidentified mechanism of $\mathrm{CD}^{+} \mathrm{T}$ cell depletion during HIV/SIV infection. Furthermore, since $\mathrm{CD}^{+} \mathrm{T}$ cell trapping by NETs persists in ART-treated SIV-infected NHPs (Figure 2C), residual NETosis may be a significant factor behind the incomplete $\mathrm{CD} 4^{+} \mathrm{T}$ cell recovery observed in HIV-infected subjects on ART.

A key unsolved aspect of HIV/SIV pathogenesis is that, in addition to the depletion of the virus targets, other immune cell subsets (i.e., $\mathrm{CD}^{+} \mathrm{T}$ cells, B cells, and even neutrophils) are also massively lost, without a clear cause. Bystander apoptosis is accepted as the main cause of death for these immune effectors (14); however, other unknown factors may be involved. We 

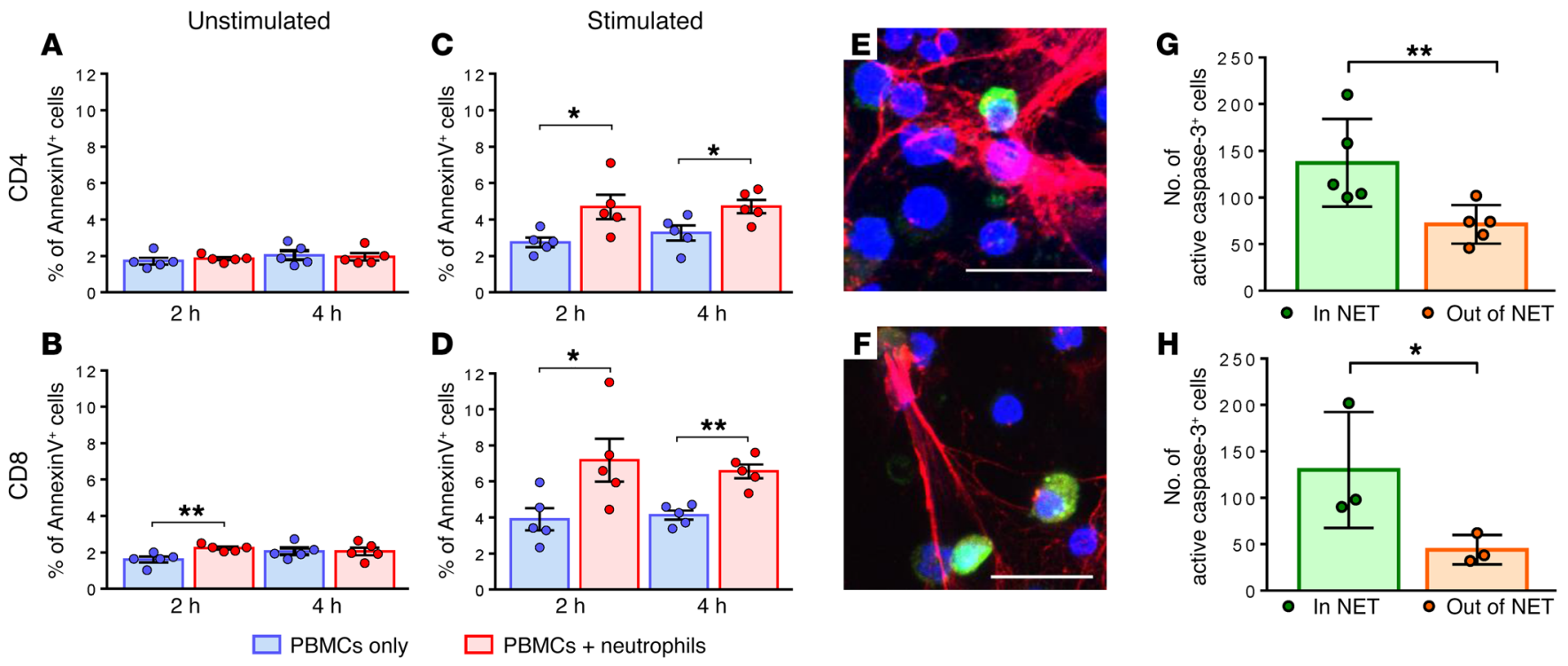

Figure 3. Cell death in NETs. No increase of CD4+ $T$ cell apoptosis (A), and minimal increase of CD8 ${ }^{+} T$ cell apoptosis (B) upon incubation with neutrophils without PMA stimulation. Significant increase of $C D 4^{+} T$ cell apoptosis (C) and CD8 $8^{+} T$ cell apoptosis (D) occurred upon incubation with neutrophils after PMA stimulation. A 2-tailed Mann-Whitney $U$ test was performed and significance was defined as ${ }^{*} P<0.05$, ${ }^{* *} P<0.01$ (A-D). Stimulated cocultured PBMCs and neutrophils from uninfected PTMs $(n=5)$ stained for active caspase-3 (green) and NE (red) showed that cells trapped in the NETs undergo apoptosis (E). Unstimulated cocultured PBMCs and PMNs from chronically infected PTMs $(n=3)$, stained for active caspase-3 (green) and NE (red), showed that cells trapped in the NETs undergo apoptosis (F). Quantification of apoptotic cells in the NET versus out of the NET in stimulated uninfected cells (G) and unstimulated infected cells (H) confirmed increased cell death in the NET. Scale bars: $20 \mu \mathrm{m}$. A 1-tailed Mann-Whitney $U$ test was performed and significance was defined as ${ }^{*} P<0.05,{ }^{* *} P<0.01(\mathbf{G}, \mathbf{H})$.

thus investigated whether NET capture is responsible for the loss of these immune cells. PBMCs and neutrophils were incubated with or without a NET stimulus and stained for CD8, CD20, or CD163, and lactoferrin. Indeed, $\mathrm{CD} 8^{+} \mathrm{T}$ cells (Figure 2D and Supplemental Figure 4), B cells (Figure 2E), and neutrophils were trapped in the NETs, similar to the $\mathrm{CD}^{+} \mathrm{T}$ cells (Figure 2, $\mathrm{A}-\mathrm{C}$ ) and monocytes (Figure 2F). The 3D confocal microscopy views clearly showed that we are dealing with true cell capture and not merely superposition of the immune cells and NETs in cultures (Supplemental Videos 1 and 2). Quantification of the cells captured by NETs failed to identify preferential targeting of a particular immune cell subset (Figure 2G). Through combined immunofluorescence for lactoferrin and RNAscope in situ hybridization with an SIVsab probe, we also showed that capture of both infected (Figure 2H, yellow arrow) and uninfected (Figure $2 \mathrm{H}$, white arrow) lymphocytes occurred in the NETs.

Our results suggest that, at least in part, bystander death of immune cells that are not directly targeted by the virus results as a pure mechanical effect of NETosis, and occurs as collateral damage rather than a targeted killing of a particular immune cell subset. Our data thus provide a plausible explanation for the loss of multiple immune cells during HIV/SIV infection, irrespective of their ability to support virus replication.

HIV infection associates a hypercoagulable state, directly linked to both a high risk of cardiovascular events and death (15). The causes of HIV-related hypercoagulability are not completely elucidated, preventing appropriate interventions to alleviate this root cause of multiple comorbidities. Since platelet trapping in the NETs may promote thrombosis (9), we posited that NETosis can lead to hypercoagulopathy in SIV/HIV infec- tion. We incubated platelets and neutrophils from SIV-infected PTMs in the presence or absence of a NET stimulus. A large number of platelets were indeed caught in the NETs (Figure 2I and Supplemental Figure 6), explaining, at least in part, the thrombocytopenia associated with SIV/HIV infection (16). Meanwhile, aggregation of platelets in the NETs (Figure 2I) may trigger thrombi formation, thus obstructing small blood vessels or complicating atherosclerotic lesions (9).

In addition to acting as mechanical barriers leading to platelet aggregation, NETs may impact coagulation through other pathways. Both neutrophils and NETs can express TF $(17,18)$, an essential activator of coagulation (19). By culturing unstimulated and stimulated neutrophils from chronically SIV-infected PTMs, we found that those generating NETs preferentially express high levels of TF (Figure 2J). The same was true for the NETs themselves (Figure 2J). In high contrast, the surrounding neutrophils were negative for TF (Figure 2J). TF expression by NETs and their ability to capture platelets could thus potentiate each other and promote an environment favorable to platelet aggregation and activation, leading to a hypercoagulable state.

To strengthen our data with more in vivo observations, we next analyzed tissues collected from chronically SIV-infected PTMs, and similar to previous studies from other research areas $(4,6)$, we found NETs (Figure 4 and Supplemental Figure 7). To accurately identify the NETs in tissues, we first assessed their presence in crypt abscesses in the gut (Figure 4A). Previous studies reported that NET density is high in pathological conditions associated with abscess formation, such as psoriasis, bronchopneumonia, and ulcerative colitis $(6,20,21)$. In tissues, NETs had a slightly different morphology than they did ex vivo: 

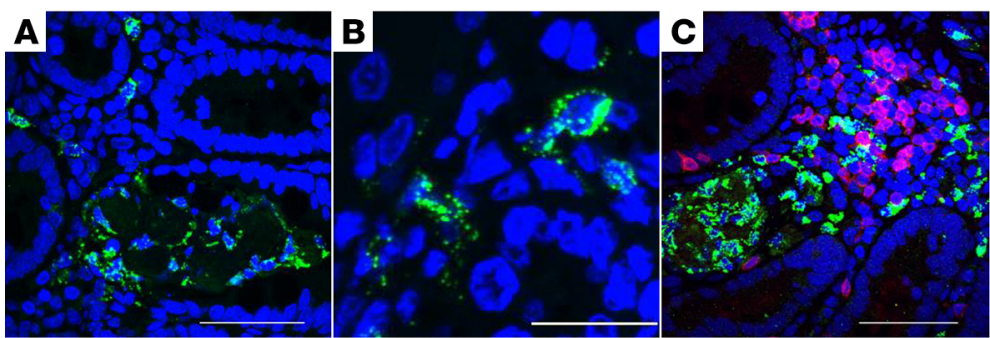

Figure 4. Assessment of NETs in tissues. In chronically SIV-infected PTMs, NETs (green) were found in crypt abscesses in the gut (A) and in liver granulomas (B). Infiltration with neutrophils that form NETs (green) able to trap CD3 ${ }^{+} \mathrm{T}$ cells (red) occurred around crypt abscesses (C). In liver granulomas, NETs (green) captured CD68+ macrophages (red) (D). In the lung, NETs (green) captured $\mathrm{CD}^{+} \mathrm{T}$ cells (red) (E). NETs (green) were found in the lamina propria of the gut distant from crypt abscesses (F); in the large vessels of the heart (C); in the glomerular capillaries in the kidney (green) (H); and appear to occlude small blood vessels in
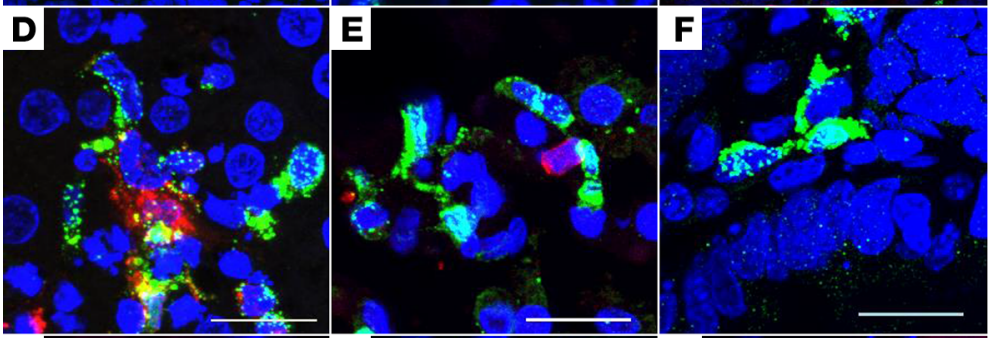
the kidney (red) (I). Scale bars: $50 \mu \mathrm{m}$ (A and C), $20 \mu \mathrm{m}$ (B, D-I). NET identification by staining for MPO (A-F, $\mathbf{H})$ and NE ( $\mathbf{G}$ and $\mathbf{I})$. Nuclear staining, DAPI (blue).
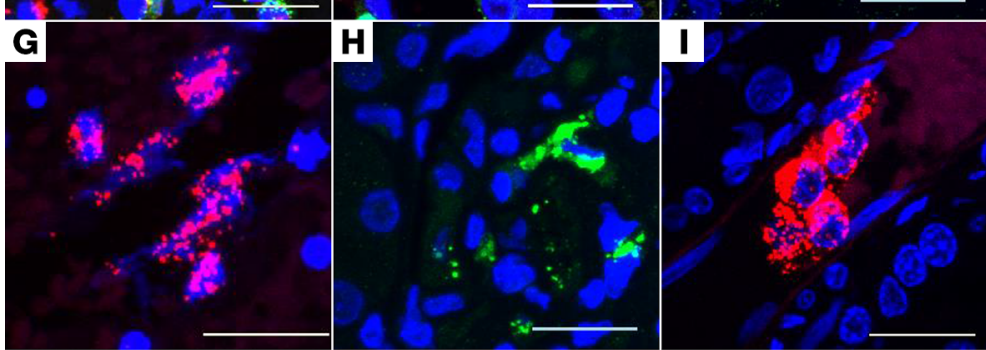

they were thicker and with a more granular, "bead on a string" appearance (22). Similar structures occurred in the liver, in the SIV-infected PTMs with liver granulomas induced by atypical Mycobacteria (Figure 4B). As described for the ex vivo-generated NETs, tissue NETs captured immune cells, such as $\mathrm{CD}^{+}$ lymphocytes and macrophages (Figure 4, C and D, and Supplemental Videos 3 and 4). Interestingly, the animals with a high frequency of crypt abscesses had the lowest CD $4^{+} \mathrm{T}$ cell counts in the gut (Supplemental Table 2). Around the crypt abscesses there were a large number of neutrophils that directly interacted with $\mathrm{T}$ cells (Figure 4C), potentially contributing to their destruction. The intensive tissue damage induced by neutrophils and their NETs may thus contribute to the severe CD4 ${ }^{+}$ $\mathrm{T}$ cell depletion and early death observed in these animals (Supplemental Table 2). The correlations between the frequency of crypt abscesses and the number of $\mathrm{CD}^{+} \mathrm{T}$ cells or survival support this hypothesis (Supplemental Figure 8). In SIV-infected PTMs, NETs were also present in the lung (Figure 4E), lamina propria of the gut, distant from crypt abscesses (Figure 4F), in blood vessels from heart (Figure $4 \mathrm{G}$ ) and kidneys, in both glomerular capillaries (Figure 4H), and in the small blood vessels outside glomeruli (Figure 4I). The T cells trapped in the NETs had morphological changes suggestive of apoptosis, such as irregular shapes and fragmented nuclei (Figure 4E and Supplemental Figure 7E). Also, in small blood vessels, the NETs and the neutrophils producing them formed small obstructive barriers (Figure 4I), supporting our ex vivo findings. These observations, together with NET ability to capture platelets (Figure 2I) and activate them via TF expression (Figure 2J), may provide a valid explanation for the high frequency of kidney microthrombi observed in chronically SIV-infected PTMs (23).
Excessive NET production during SIV infection may thus provide a dual mechanism for enhanced thrombi formation in the context of low platelet counts. NETosis might thus decisively contribute to both the high risk of cardiovascular events observed in HIV/SIV-infected subjects/NHPs $(15,23)$, and to the development of thrombotic microangiopathy, which may be at the origin of multiple HIV-related comorbidities.

Altogether, our results point to a new paradigm of SIV/HIV pathogenesis, in which neutrophils attempting to phagocytize translocated microbes are overwhelmed and driven to excessive suicidal NET formation. The beneficial effects of NETs, such as the elimination of free virions and of HIV-infected $\mathrm{CD} 4^{+} \mathrm{T}$ cells, are then gradually and largely outweighed by multiple collateral damages, such as indiscriminate trapping and destruction of immune cells in the NETs, and excessive platelet capture and aggregation. Excessive NETosis characteristic to HIV infection can thus contribute to immune failure after ART, and to the development of both non-HIV-associated comorbidities and end-stage organ disease characteristic to SIV/HIV infection. Adjuvant therapies to eliminate NETs may thus be beneficial for HIV-infected patients.

\section{Methods}

For a complete description of the methods, see Supplemental Methods.

Study approval. The animals were housed at the Plum Borough Research Center, University of Pittsburgh, where they were monitored as per the Association for Assessment and Accreditation of Laboratory Animal Care (AAALAC) International, and the NIH Guide for the Care and Use of Laboratory Animals (National Academies Press, 2011). The study was approved by the IACUC of the University of Pittsburgh (protocols 15045829, 17040178, 0911844, 0907039, 12121250, 12040408). 


\section{Author contributions}

RS, EBC, CA, and IP designed these studies. RS, EBC, and SMKV conducted the experiments. RS, EBC, EF, NK, and SMKV acquired the data. RS, EBC, NK, CA, and IP analyzed and interpreted the data. RMR performed advanced data analyses. RS, EBC, RMR, CA, and IP wrote the manuscript.

\section{Acknowledgments}

We thank Simon Watkins (Center for Biologic Imaging, University of Pittsburgh) for providing instrumentation (grant 1S10OD019973-01). This work was supported by the National
Heart, Lung, and Blood Institute/National Institute of Diabetes and Digestive and Kidney Diseases/National Institute of Allergy and Infectious Diseases R01 grants HL117715 and HL123096 (to IP), DK113919 (to IP and CA), AI119346 (to CA), and RR025781 (to CA and IP). The funders had no role in study design, data collection and analysis, decision to publish, or preparation of the manuscript.

Address correspondence to: Ivona Pandrea, 9045 BST3, 3501 Fifth Avenue, Pittsburgh, Pennsylvania 15261, USA. Phone: 412.624.3242; Email:pandrea@pitt.edu.
1. Brinkmann V, et al. Neutrophil extracellular traps kill bacteria. Science (New York, NY). 2004;303(5663):1532-1535.

2. Galani IE, Andreakos E. Neutrophils in viral infections: Current concepts and caveats. JLeukoc Biol. 2015;98(4):557-564.

3. Fuchs TA, et al. Novel cell death program leads to neutrophil extracellular traps. J Cell Biol. 2007;176(2):231-241.

4. Yipp BG, et al. Infection-induced NETosis is a dynamic process involving neutrophil multitasking in vivo. Nat Med. 2012;18(9):1386-1393.

5. Urban CF, et al. Neutrophil extracellular traps contain calprotectin, a cytosolic protein complex involved in host defense against Candida albicans. PLoS Pathog. 2009;5(10):e1000639.

6. Savchenko AS, et al. Long pentraxin 3 (PTX3) expression and release by neutrophils in vitro and in ulcerative colitis. Pathol Int. 2011;61(5):290-297.

7. de Buhr N, von Köckritz-Blickwede M. How neutrophil extracellular traps become visible. J Immunol Res. 2016;2016:4604713.

8. Saitoh T, et al. Neutrophil extracellular traps mediate a host defense response to human immunodeficiency virus-1. Cell Host Microbe. 2012;12(1):109-116.

9. Fuchs TA, et al. Extracellular DNA traps pro- mote thrombosis. Proc Natl Acad Sci U S A. 2010;107(36):15880-15885.

10. Jorch SK, Kubes P. An emerging role for neutrophil extracellular traps in noninfectious disease. Nat Med. 2017;23(3):279-287.

11. Kristoff J, et al. Early microbial translocation blockade reduces SIV-mediated inflammation and viral replication. JClin Invest. 2014;124(6):2802-2806.

12. Brenchley JM, et al. Microbial translocation is a cause of systemic immune activation in chronic HIV infection. Nat Med. 2006;12(12):1365-1371.

13. Sayah DM, et al. Neutrophil extracellular traps are pathogenic in primary graft dysfunction after lung transplantation. Am J Respir Crit Care Med. 2015;191(4):455-463.

14. Finkel TH, et al. Apoptosis occurs predominantly in bystander cells and not in productively infected cells of HIV- and SIV-infected lymph nodes. Nat Med. 1995;1(2):129-134.

15. Kuller LH, et al. Inflammatory and coagulation biomarkers and mortality in patients with HIV infection. PLoS Med. 2008;5(10):e203.

16. Scaradavou A. HIV-related thrombocytopenia. Blood Rev. 2002;16(1):73-76.

17. Stakos DA, et al. Expression of functional tissue factor by neutrophil extracellular traps in culprit artery of acute myocardial infarction. Eur Heart J. 2015;36(22):1405-1414.

18. Darbousset R, et al. Tissue factor-positive neutrophils bind to injured endothelial wall and initiate thrombus formation. Blood. 2012;120(10):2133-2143.

19. Schechter ME, et al. Inflammatory monocytes expressing tissue factor drive SIV and HIV coagulopathy. Sci Transl Med. 2017;9(405):eaam5441.

20. Hu SC, Yu HS, Yen FL, Lin CL, Chen GS, Lan CC. Neutrophil extracellular trap formation is increased in psoriasis and induces human $\beta$-defensin-2 production in epidermal keratinocytes. Sci Rep. 2016;6:31119.

21. Shiogama K, Onouchi T, Mizutani Y, Sakurai K, Inada K, Tsutsumi Y. Visualization of neutrophil extracellular traps and fibrin meshwork in human fibrinopurulent inflammatory lesions: I. Light microscopic study. Acta Histochem Cytochem. 2016;49(4):109-116.

22. Obermayer A, et al. New aspects on the structure of neutrophil extracellular traps from chronic obstructive pulmonary disease and in vitro generation. PLoS One. 2014;9(5):e97784.

23. Pandrea I, et al. Coagulation biomarkers predict disease progression in SIV-infected nonhuman primates. Blood. 2012;120(7):1357-1366. 